\title{
Czechoslovak Mathematical Journal
}

\section{Judita Lihová}

On posets with isomorphic interval posets

Czechoslovak Mathematical Journal, Vol. 49 (1999), No. 1, 67-80

Persistent URL: http://dml.cz/dmlcz/127468

\section{Terms of use:}

(C) Institute of Mathematics AS CR, 1999

Institute of Mathematics of the Czech Academy of Sciences provides access to digitized documents strictly for personal use. Each copy of any part of this document must contain these Terms of use.

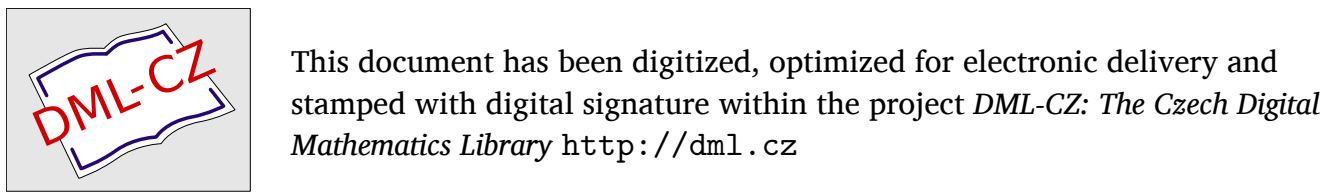




\title{
ON POSETS WITH ISOMORPHIC INTERVAL POSETS
}

\author{
Judita Lihová, Košice
}

(Received April 4, 1996)

Let $\mathbb{A}=(A, \leqslant)$ be a partially ordered set, Int $\mathbb{A}$ the system of all (nonempty) intervals of $\mathbb{A}$, partially ordered by the set-theoretical inclusion $\subseteq$. We are interested in partially ordered sets $\mathbb{B}=(B, \leqslant)$ with Int $\mathbb{B}$ isomorphic to Int $\mathbb{A}$. We are going to show that they correspond to couples of binary relations on $A$ satisfying some conditions. If $\mathbb{A}$ is a directed partially ordered set, the only $\mathbb{B}$ with Int $\mathbb{B}$ isomorphic to Int $\mathbb{A}$ are $\mathbb{A}_{1}^{\delta} \times \mathbb{A}_{2}$ corresponding to direct decompositions $\mathbb{A}_{1} \times \mathbb{A}_{2}$ of $\mathbb{A}\left(\mathbb{A}_{1}^{\delta}\right.$ denotes the dual of $\left.\mathbb{A}_{1}\right)$. The present results include those presented in the paper [11] by $\mathrm{V}$. Slavík. Systems of intervals, particularly of lattices, have been investigated by many authors, cf. [1]-[11].

By an interval of a partially ordered set $\mathbb{A}=(A, \leqslant)$ a set $\langle a, b\rangle=\{x \in A: a \leqslant x \leqslant$ $b\}$ with $a, b \in A, a \leqslant b$ is meant. If $a=b$, we use the notation $\langle a\rangle$ instead of $\langle a, a\rangle$. The system of all intervals of $\mathbb{A}$ is denoted by Int $\mathbb{A}$. Consider the set-theoretical inclusion on Int $\mathbb{A}$. The following lemma is easy to verify:

1.1. Lemma. a) $\langle a, b\rangle=\inf \left\{\left\langle a_{1}, b_{1}\right\rangle,\left\langle a_{2}, b_{2}\right\rangle\right\}$ if and only if $\langle a, b\rangle=\left\langle a_{1}, b_{1}\right\rangle \cap$ $\left\langle a_{2}, b_{2}\right\rangle$;

b) $\langle a, b\rangle=\sup \left\{\left\langle a_{1}, b_{1}\right\rangle,\left\langle a_{2}, b_{2}\right\rangle\right\}$ if and only if $a=\inf \left\{a_{1}, a_{2}\right\}, b=\sup \left\{b_{1}, b_{2}\right\}$.

Let $U, V$ be binary relations on $A$. Consider the following conditions:

(P1) $U, V \subseteq\{(x, y) \in A \times A: x \nmid y\}$;

(P2) $x, y \in A, x \leqslant y \Longrightarrow$ there exists a unique couple of elements $p, q \in\langle x, y\rangle$ satisfying $p V x U q V y U p$;

(P3) $u \leqslant x, y, x V u U y \Longrightarrow u=\inf \{x, y\}, v=\sup \{x, y\}$ exists and $y V v U x$ holds;

$\left(\mathrm{P} 3^{\prime}\right) v \geqslant x, y, y V v U x \Longrightarrow v=\sup \{x, y\}, u=\inf \{x, y\}$ exists and $x V u U y$ holds; 
(P4) $a=a_{1} U a_{2} U \ldots U a_{n}=a^{\prime}, a=a_{1}^{\prime} V a_{2}^{\prime} V \ldots V a_{m}^{\prime}=a^{\prime}(n, m \in N) \Longrightarrow a=a^{\prime}$;

(P5) for every $a, a^{\prime} \in A$ there exist $n, m \in N, a_{1}, \ldots, a_{n}, a_{1}^{\prime}, \ldots, a_{m}^{\prime} \in A$ satisfying $a=a_{1} U a_{2} U \ldots U a_{n}=a_{1}^{\prime} V a_{2}^{\prime} V \ldots V a_{m}^{\prime}=a^{\prime}$.

We are going to prove the following theorem:

1.2. Theorem. Let $\mathbb{A}$ be a connected partially ordered set. Then there exists a mapping $\Phi$ of the system of all couples of binary relations $U, V$ on $A$ satisfying the conditions $(\mathrm{P} 1)-\left(\mathrm{P} 3^{\prime}\right)$ onto the system of all isomorphism classes of partially ordered sets $\mathbb{B}$ with Int $\mathbb{B}$ isomorphic to Int $\mathbb{A}$. If a couple $(U, V)$ satisfies $(\mathrm{P} 1)-(\mathrm{P} 5)$, then the class $\Phi((U, V))$ consists of all partially ordered sets isomorphic to $\mathbb{A}_{1}^{\delta} \times \mathbb{A}_{2}$ for a direct decomposition $\mathbb{A}_{1} \times \mathbb{A}_{2}$ of $\mathbb{A}$. Conversely, the class of all partially ordered sets isomorphic to $\mathbb{A}_{1}^{\delta} \times \mathbb{A}_{2}$ for a direct decomposition $\mathbb{A}_{1} \times \mathbb{A}_{2}$ of $\mathbb{A}$ is $\Phi((U, V))$ for a couple $(U, V)$ satisfying $(\mathrm{P} 1)-(\mathrm{P} 5)$.

Let us remark that the connectivity of $\mathbb{A}$ is not a limiting assumption. Namely, if $\mathbb{P}$ is any partially ordered set, $P$ can be decomposed into maximal connected subsets $P_{i}(i \in I)$ and the system Int $\mathbb{P}$ is the cardinal sum of the interval posets Int $\mathbb{P}_{i}$ of these subsets. Now a partially ordered set $\mathbb{Q}$ satisfies the condition Int $\mathbb{Q} \cong$ Int $\mathbb{P}$ if and only if $\mathbb{Q}$ is the cardinal sum of some $\mathbb{Q}_{i}(i \in I)$ with Int $\mathbb{Q}_{i} \cong \operatorname{Int} \mathbb{P}_{i}$.

Further let us notice that if partially ordered sets $\mathbb{A}, \mathbb{B}$ have isomorphic interval posets, then they are of the same cardinality; so we may assume, without loss of generality, that $\mathbb{A}, \mathbb{B}$ have the same underlying set.

2 .

Let $\mathbb{A}=(A, \leqslant)$ be a connected partially ordered set, $U, V$ binary relations on $A$ satisfying $(\mathrm{P} 1)-\left(\mathrm{P} 3^{\prime}\right)$. First we will show some properties of $U, V$ following from the conditions $(\mathrm{P} 1)-\left(\mathrm{P} 3^{\prime}\right)$.

The following is obtained immediately, using (P2).

2.1. Lemma. The relations $U, V$ are reflexive.

2.2. Lemma. The relations $U, V$ are symmetric.

Pro of. Let $x U y$. By (P1) $x, y$ are comparable. Suppose, e.g., that $x \leqslant y$. We have $x \leqslant x, y, x V x U y$ and since $y=\sup \{x, y\}$, using (P3) we obtain $y U x$. To prove $y U x$ for $x \geqslant y$, we use $\left(\mathrm{P} 3^{\prime}\right)$.

2.3. Lemma. If $x, y \in A$ and one of these elements covers the other, then $(x, y) \in U \cup V$. 
This follows immediately from (P2).

2.4. Lemma. If $(x, y) \in U \cap V$, then $x=y$.

Proof. Let $(x, y) \in U \cap V$. Without loss of generality we can suppose $x \leqslant y$. Then both $x U y V y$ and $x U x V y$ hold, so $x=y$ by (P2).

2.5. Lemma. If $x \leqslant y \leqslant z$, then $x U y U z$ implies $x U z$ and $x V y V z$ implies $x V z$.

P r o of. We are going to prove, e.g., the part concerning $U$. Hence let $x \leqslant y \leqslant z$, $x U y U z$. (P2) ensures the existence of an element $p \in\langle x, z\rangle$ with $z U p V x$. Now $x \leqslant p, y, p V x U y$, so that $\sup \{p, y\}=v$ exists and satisfies $y V v U p$ by (P3). Evidently $v \leqslant z$. We have $y \leqslant v, z, v V y U z$, so in view of (P3) we obtain $y=\inf \{v, z\}=v$. But then $x=\inf \{p, y\}=p$ and consequently $x U z$.

2.6. Lemma. Let $x, y \in A, x \leqslant y, p, q$ be as in (P2). If $a \in\langle x, y\rangle$, there exists a unique quadruple of elements $p_{1} \in\langle x, p\rangle, q_{1} \in\langle x, q\rangle, p_{2} \in\langle p, y\rangle, q_{2} \in\langle q, y\rangle$ satisfying $a U p_{1} V x U q_{1} V a U q_{2} V y U p_{2} V a, p_{1} V p U p_{2}, q_{1} U q V q_{2}$.

Proof. Let $a \in\langle x, y\rangle$. Then $x \leqslant a$ implies the existence of $p_{1}, q_{1} \in\langle x, a\rangle$ satisfying $p_{1} V x U q_{1} V a U p_{1}$ and $a \leqslant y$ implies that $p_{2} V a U q_{2} V y U p_{2}$ for some $p_{2}, q_{2} \in$ $\langle a, y\rangle$, by (P2). Using again (P2) we obtain that there exist $p^{\prime} \in\left\langle p_{1}, p_{2}\right\rangle, q^{\prime} \in\left\langle q_{1}, q_{2}\right\rangle$ such that $p_{1} V p^{\prime} U p_{2}, q_{1} U q^{\prime} V q_{2}$. But then 2.5 yields $p^{\prime} V x U q^{\prime} V y U p^{\prime}$. The uniqueness of $p, q$ in (P2) implies $p^{\prime}=p, q^{\prime}=q$. The uniqueness of $p_{1}, q_{1}, p_{2}, q_{2}$ follows from (P3) and $\left(\mathrm{P} 3^{\prime}\right)$. Namely, $p_{1}=\inf \{p, a\}, q_{1}=\inf \{a, q\}, p_{2}=\sup \{p, a\}, q_{2}=\sup \{a, q\}$.

2.7. Lemma. If $x \leqslant a \leqslant y$, then $x U y$ implies $x U a U y$ and $x V y$ implies $x V a V y$.

Proof. Let $x \leqslant a \leqslant y, x U y$. Using the notation as in 2.6, we have $p=x$, $q=y, p_{1}=x, q_{1}=a, p_{2}=a, q_{2}=y$. By 2.6 we have $p U p_{2} U y$, hence $x U a U y$. The part concerning $V$ can be shown analogously.

2.8. Lemma. Let $x, y \in A, x \leqslant y, p, q$ be as in (P2). Then for each $a \in\langle x, y\rangle$, $\inf \{p, a\}, \inf \{a, q\}$ exist and they satisfy $p V \inf \{p, a\} U a V \inf \{a, q\} U q$. The mapping $\alpha: a \mapsto(\inf \{p, a\}, \inf \{a, q\})$ is an isomorphism of $\langle x, y\rangle$ onto $\langle x, p\rangle \times\langle x, q\rangle$.

Proof. Let $a \in\langle x, y\rangle, p_{1}, q_{1}$ be as in 2.6. Then $p_{1}=\inf \{p, a\}, q_{1}=\inf \{a, q\}$ by (P3). Further, 2.6 ensures that $p V p_{1} U a V q_{1} U q$ holds. Now using (P3') and 2.6 we obtain $a=\sup \left\{p_{1}, q_{1}\right\}$. Let $p_{1}^{\prime} \in\langle x, p\rangle, q_{1}^{\prime} \in\langle x, q\rangle$. Since $x \leqslant p_{1}^{\prime}, q_{1}^{\prime}$ and $p_{1}^{\prime} V x U q_{1}^{\prime}$ holds, by 2.7, the condition (P3) yields that $\sup \left\{p_{1}^{\prime}, q_{1}^{\prime}\right\}=a^{\prime}$ exists and we have $q_{1}^{\prime} V a^{\prime} U p_{1}^{\prime}$. But then $p_{1}^{\prime}=\inf \left\{p, a^{\prime}\right\}, q_{1}^{\prime}=\inf \left\{a^{\prime}, q\right\}$, so that $\alpha\left(a^{\prime}\right)=\left(p_{1}^{\prime}, q_{1}^{\prime}\right)$. We have proved that $\alpha$ is onto. 
Let $a, a^{\prime} \in\langle x, y\rangle, a \leqslant a^{\prime}$. Then evidently $(\inf \{p, a\}, \inf \{a, q\}) \leqslant\left(\inf \left\{p, a^{\prime}\right\}\right.$, $\left.\inf \left\{a^{\prime}, q\right\}\right)$. Hence $\alpha$ preserves the order.

Finally, let $a, a^{\prime} \in\langle x, y\rangle,(\inf \{p, a\}, \inf \{a, q\}) \leqslant\left(\inf \left\{p, a^{\prime}\right\}, \inf \left\{a^{\prime}, q\right\}\right)$. Then $a=\sup \{\inf \{p, a\}, \inf \{a, q\}\} \leqslant \sup \left\{\inf \left\{p, a^{\prime}\right\}, \inf \left\{a^{\prime}, q\right\}\right\}=a^{\prime}$, completing the proof.

2.9. Lemma. Let $x, y \in A, x \leqslant y, p, q$ be as in (P2). If $x \leqslant a \leqslant a^{\prime} \leqslant y$ and $a U a^{\prime}\left(a V a^{\prime}\right)$, then $\inf \{p, a\}=\inf \left\{p, a^{\prime}\right\}\left(\inf \{a, q\}=\inf \left\{a^{\prime}, q\right\}\right)$.

Proof. Suppose that $x \leqslant a \leqslant a^{\prime} \leqslant y$ and, e.g., $a U a^{\prime}$. Using 2.8 we get $\inf \{p, a\} \leqslant \inf \left\{p, a^{\prime}\right\}, p V \inf \{p, a\} U a U a^{\prime}$. Now $\inf \left\{p, a^{\prime}\right\} \in\left\langle\inf \{p, a\}, a^{\prime}\right\rangle$, so that $\inf \{p, a\} U \inf \left\{p, a^{\prime}\right\}$. But simultaneously $\inf \{p, a\} V \inf \left\{p, a^{\prime}\right\}$ by 2.7. Hence $\inf \{p, a\}=\inf \left\{p, a^{\prime}\right\}$ by 2.4 .

Now we are going to introduce a "new" order on $A$, corresponding to a couple of $U, V$ satisfying $(\mathrm{P} 1)-\left(\mathrm{P} 3^{\prime}\right)$.

2.10. Definition. For $x, y \in A$ set $x \leqslant 1$ y if there exists $u \in A, u \leqslant x, y$, satisfying $x V u U y$.

2.11. Lemma. The above defined relation $\leqslant_{1}$ is a partial order.

Proof. The reflexivity of $U, V$ ensures that $x \leqslant 1 \quad x$ for each $x \in A$. Let $x \leqslant 1 y, y \leqslant 1 x$. Then there exist $u_{1}, u_{2}$ such that $u_{1} \leqslant x, y, x V u_{1} U y, u_{2} \leqslant y, x$, $y V u_{2} U x$. Using (P3) we obtain $u_{1}=\inf \{x, y\}=u_{2}$. Hence $\left(u_{1}, x\right) \in U \cap V$ and also $\left(u_{1}, y\right) \in U \cap V$ and consequently $x=u_{1}=y$ by 2.4. Let $x \leqslant_{1} y, y \leqslant_{1} z$. Then there exist $u_{1}, u_{2} \in A$ satisfying $u_{1} \leqslant x, y, x V u_{1} U y, u_{2} \leqslant y, z, y V u_{2} U z$. Using (P3') we obtain that $\inf \left\{u_{1}, u_{2}\right\}=u$ exists and $u_{1} V u U u_{2}$ holds. But then $u \leqslant x, z$ and $x V u U z$ by 2.5 , so that $x \leqslant 1 z$.

The aim is to prove that $\operatorname{Int}(A, \leqslant) \cong \operatorname{Int}(A, \leqslant 1)$. Let $x, y \in A, x \leqslant y, p, q$ be as in (P2). Then evidently $p \leqslant_{1} q$. Set $f(\langle x, y\rangle)=\langle p, q\rangle_{1}$, where $\langle p, q\rangle_{1}=\left\{t \in A: p \leqslant_{1}\right.$ $\left.t \leqslant_{1} q\right\}$. Recall that $\langle x, y\rangle$ is isomorphic to $\langle x, p\rangle \times\langle x, q\rangle$. Now we have:

2.12. Lemma. The mapping $\alpha$ defined in 2.8 is an isomorphism of $\langle p, q\rangle_{1}$ onto $\langle x, p\rangle^{\delta} \times\langle x, q\rangle$.

Proof. Evidently $a \in\langle x, y\rangle$ if and only if $a \in\langle p, q\rangle_{1}$ and $\alpha$ is onto. Further let us suppose that $a, a^{\prime} \in\langle p, q\rangle_{1}, a \leqslant 1 a^{\prime}$. We have to $\operatorname{prove} \inf \{p, a\} \geqslant \inf \left\{p, a^{\prime}\right\}$, $\inf \{a, q\} \leqslant \inf \left\{a^{\prime}, q\right\}$. Take $p_{1}=\inf \{p, a\}, q_{1}^{\prime}=\inf \left\{a^{\prime}, q\right\}$ and $u \leqslant a, a^{\prime}$ satisfying $a V u U a^{\prime}$. In view of $\left(\mathrm{P}^{\prime}\right), r=\inf \left\{p_{1}, u\right\}, s=\inf \left\{u, q_{1}^{\prime}\right\}$ exist such that $p_{1} V r U u V s U q_{1}^{\prime}$. But then $p V r U a^{\prime}, a V s U q$, so that $r=\inf \left\{p, a^{\prime}\right\}, s=\inf \{a, q\}$ 
and we have $r \leqslant p_{1}, s \leqslant q_{1}^{\prime}$. Conversely let $a, a^{\prime} \in\langle p, q\rangle_{1}, p_{1} \geqslant p_{1}^{\prime}, q_{1} \leqslant q_{1}^{\prime}$, where $p_{1}=\inf \{p, a\}, q_{1}=\inf \{a, q\}, p_{1}^{\prime}=\inf \left\{p, a^{\prime}\right\}, q_{1}^{\prime}=\inf \left\{a^{\prime}, q\right\}$. Since $x \leqslant p_{1}^{\prime}, q_{1}$ and $p_{1}^{\prime} V x U q_{1}, \sup \left\{p_{1}^{\prime}, q_{1}\right\}=t$ exists and $q_{1} V t U p_{1}^{\prime}$. Obviously $t \leqslant a, a^{\prime}$. Moreover, $a V q_{1}$ yields $a V t$ and $p_{1}^{\prime} U a^{\prime}$ implies $t U a^{\prime}$. Thus $a \leqslant_{1} a^{\prime}$. The proof is complete.

2.13. Lemma. The mapping $f$ assigning to $\langle x, y\rangle$ the interval $\langle p, q\rangle_{1}$ is an isomorphism of $\operatorname{Int}(A, \leqslant)$ onto $\operatorname{Int}(A, \leqslant 1)$.

Proof. Let $r \leqslant_{1} s$. Then there exists $u \leqslant r, s$ such that $r V u U s$. By (P3), $v=\sup \{r, s\}$ exists and $s V v U r$ holds. Evidently $f(\langle u, v\rangle)=\langle r, s\rangle_{1}$. The mapping $f$ is onto.

Now let $\langle x, y\rangle \subseteq\left\langle x_{1}, y_{1}\right\rangle, f(\langle x, y\rangle)=\langle p, q\rangle_{1}, f\left(\left\langle x_{1}, y_{1}\right\rangle\right)=\left\langle p_{1}, q_{1}\right\rangle_{1}$. Take $\inf \left\{p_{1}, p\right\}=p_{1}^{\prime}, \inf \left\{q, q_{1}\right\}=q_{1}^{\prime}$. We have $p_{1}^{\prime} \leqslant p_{1}, p, p_{1} V p_{1}^{\prime} U p$, so $p_{1} \leqslant 1 p$. Analogously $q_{1}^{\prime} \leqslant q, q_{1}, q V q_{1}^{\prime} U q_{1}$ ensures $q \leqslant 1 q_{1}$. Hence $\langle p, q\rangle_{1} \subseteq\left\langle p_{1}, q_{1}\right\rangle_{1}$.

Next suppose that $f(\langle x, y\rangle)=\langle p, q\rangle_{1} \subseteq\left\langle p_{1}, q_{1}\right\rangle_{1}=f\left(\left\langle x_{1}, y_{1}\right\rangle\right)$. We have to show $\langle x, y\rangle \subseteq\left\langle x_{1}, y_{1}\right\rangle$. Let $u \leqslant p_{1}, p, p_{1} V u U p$ and $v \leqslant q, q_{1}, q V v U q_{1}$. Since $p \geqslant u, x$, $x V p U u$ and $q \geqslant x, v, v V q U x$, there exist $a \leqslant u, x, b \leqslant x, v$ satisfying $u V a U x V b U v$, by $\left(\mathrm{P} 3^{\prime}\right)$. Finally consider $c=\inf \{a, b\}$, whose existence follows from $\left(\mathrm{P} 3^{\prime}\right)$. We have $p_{1} V u V a V c U b U v U q_{1}$, hence $c=\inf \left\{p_{1}, q_{1}\right\}=x_{1}$ by (P3) and (P2). Now obviously $x_{1} \leqslant x$. The relation $y \leqslant y_{1}$ can be proved analogously.

Summarizing, we have:

2.14. Theorem. Let $\mathbb{A}=(A, \leqslant)$ be a connected partially ordered set, $U, V$ binary relations on $A$ satisfying $(\mathrm{P} 1)-\left(\mathrm{P}^{\prime}\right)$. If $\leqslant_{1}$ is the relation on $A$ defined as in 2.10 with the aid of $U, V$, then $(A, \leqslant 1)$ is a partially ordered set with $\operatorname{Int}\left(A, \leqslant_{1}\right)$ isomorphic to $\operatorname{Int}(A, \leqslant)$.

It is easy to see that the couples $U_{1}=\{(x, y) \in A \times A: x \nmid y\}, V_{1}=\{(x, x)$ : $x \in A\}$ and $U_{2}=\{(x, x): x \in A\}, V_{2}=\{(x, y) \in A \times A: x \nmid y\}$ satisfy the conditions $(\mathrm{P} 1)-\left(\mathrm{P} 3^{\prime}\right)$. The corresponding orders $\leqslant_{1}, \leqslant_{2}$ are $\leqslant$ and $\leqslant^{\delta}$, respectively. Some partially ordered sets $\mathbb{A}=(A, \leqslant)$ have no other orders $\leqslant 1$ besides $\leqslant$ and $\leqslant^{\delta}$, satisfying Int $(A, \leqslant 1) \cong \operatorname{Int}(A, \leqslant)$. This is the case e.g. for $\mathbb{A}$ in Fig. 1 . On the other hand, it is easy to see that the partially ordered sets in Fig. 2 and Fig. 3 have isomorphic interval systems, but they are neither isomorphic nor dually isomorphic. In fact, the first is the direct product of two copies of $\mathbb{A}$ in Fig. 1, while the other is isomorphic to $\mathbb{A}^{\delta} \times \mathbb{A}$.

Further assume that $U, V$ satisfy also the conditions (P4), (P5). Define binary relations $\bar{U}, \bar{V}$ on $A$ as follows: 


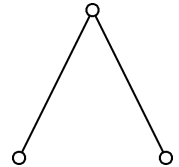

Fig. 1

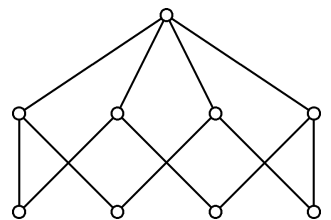

Fig. 2

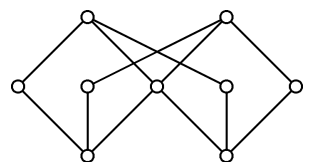

Fig. 3

2.15. Definition. For $x, y \in A$ set $x \bar{U} y(x \bar{V} y)$ if there exists a finite sequence $x_{1}, x_{2}, \ldots, x_{n}$ of elements of $A$ such that $x_{1}=x, x_{n}=y$ and every two adjoining elements are in the relation $U(V)$.

The following statement is evident.

2.16. Lemma. The relations $\bar{U}, \bar{V}$ are equivalence relations.

Consider the decompositions $A / \bar{U}, A / \bar{V}$. Denote by $[a] \bar{U},[a] \bar{V}$ the equivalence classes containing the element $a$.

2.17. Definition. Set $[a] \bar{U} \leqslant[b] \bar{U}([a] \bar{V} \leqslant[b] \bar{V})$ if and only if there exist $a_{1} \in[a] \bar{U}, b_{1} \in[b] \bar{U}\left(a_{1} \in[a] \bar{V}, b_{1} \in[b] \bar{V}\right)$ satisfying $a_{1} \leqslant b_{1}$.

2.18. Lemma. For any $a, b \in A$ the following conditions are equivalent:

(1) $[a] \bar{U} \leqslant[b] \bar{U}$;

(2) for each $a_{1} \in[a] \bar{U}$ there exists $b_{1} \in[b] \bar{U}$ with $a_{1} \leqslant b_{1}$;

(3) for each $b_{1} \in[b] \bar{U}$ there exists $a_{1} \in[a] \bar{U}$ with $a_{1} \leqslant b_{1}$.

Pro of. The implications $(2) \Longrightarrow(1),(3) \Longrightarrow(1)$ are evident. We are going to prove $(1) \Longrightarrow(2)$. The proof of $(1) \Longrightarrow(3)$ would be analogous. So let $[a] \bar{U} \leqslant[b] \bar{U}$. We can suppose that $a \leqslant b$. Take any $a_{1} \in[a] \bar{U}$. Then there exist $x_{1}, \ldots, x_{n}$ such that $a=x_{1}, a_{1}=x_{n}, x_{1} \leqslant x_{2}, x_{2} \geqslant x_{3}, \ldots, x_{n-1} \geqslant x_{n}, x_{1} U x_{2} U \ldots U x_{n}$. Using the conditions (P2), (P3) we can construct elements $y_{1}, y_{2}, \ldots, y_{n}$ such that $y_{1} \in\left\langle x_{1}, b\right\rangle$, $x_{1} V y_{1} U b, y_{2} \geqslant y_{1}, x_{2}, x_{2} V y_{2} U y_{1}, y_{3} \in\left\langle x_{3}, y_{2}\right\rangle, x_{3} V y_{3} U y_{2}, \ldots, y_{n} \in\left\langle x_{n}, y_{n-1}\right\rangle$, $x_{n} V y_{n} U y_{n-1}$. We have $a_{1} \leqslant y_{n}, y_{n} \in[b] \bar{U}$.

Obviously the same holds for $\bar{V}$.

2.19. Lemma. The above defined relation $\leqslant$ on $A / \bar{U}$ is a partial order.

Proof. The reflexivity is trivial. Further let $[a] \bar{U} \leqslant[b] \bar{U},[b] \bar{U} \leqslant[a] \bar{U}$. Then there exist $a_{1}, a_{2} \in[a] \bar{U}$ satisfying $a_{1} \leqslant b \leqslant a_{2}$. Take $z \in\left\langle a_{1}, a_{2}\right\rangle$ such that $a_{1} U z V a_{2}$. We have $z \bar{U} a_{2}$ and simultaneously $z V a_{2}$. Using (P4) we obtain $z=a_{2}$ and consequently $a_{1} \leqslant b \leqslant z$, which implies $a_{1} U b$ by 2.7 . Hence $[b] \bar{U}=\left[a_{1}\right] \bar{U}=[a] \bar{U}$. Finally, let $[a] \bar{U} \leqslant[b] \bar{U},[b] \bar{U} \leqslant[c] \bar{U}$. Then there exist $a_{1} \in[a] \bar{U}, c_{1} \in[c] \bar{U}$ such that $a_{1} \leqslant b \leqslant c_{1}$ and this implies $[a] \bar{U} \leqslant[c] \bar{U}$. 
Evidently the same holds for $\bar{V}$. The symbol $\mathbb{A} / \bar{U}(\mathbb{A} / \bar{V})$ will be used for $A / \bar{U}$ $(A / \bar{V})$ with the order $\leqslant$ as above.

2.20. Theorem. Let $\mathbb{A}=(A, \leqslant)$ be a connected partially ordered set, $U, V$ binary relations on $A$ satisfying $(\mathrm{P} 1)-(\mathrm{P} 5)$. If $\leqslant_{1}$ is as in 2.10 , then $\mathbb{A}$ is isomorphic to $\mathbb{A} / \bar{U} \times \mathbb{A} / \bar{V}$, while $\mathbb{A}_{1}=\left(A, \leqslant_{1}\right)$ is isomorphic to $(\mathbb{A} / \bar{U})^{\delta} \times \mathbb{A} / \bar{V}$.

Proof. Define $\alpha: A \rightarrow A / \bar{U} \times A / \bar{V}$ by $\alpha(a)=([a] \bar{U},[a] \bar{V}) . \quad \alpha$ is onto: Take $\left(\left[a_{1}\right] \bar{U},\left[a_{2}\right] \bar{V}\right) \in A / \bar{U} \times A / \bar{V}$. By (P5) there exists $x \in A$ satisfying $a_{1} \bar{U} x \bar{V} a_{2}$. Then $\alpha(x)=\left(\left[a_{1}\right] \bar{U},\left[a_{2}\right] \bar{V}\right)$.

The implication $a \leqslant b \Longrightarrow \alpha(a) \leqslant \alpha(b)$ is evident. Conversely, let $\alpha(a) \leqslant \alpha(b)$. Then $[a] \bar{U} \leqslant[b] \bar{U},[a] \bar{V} \leqslant[b] \bar{V}$ and consequently $a \leqslant b_{1}, b_{2}$ for some $b_{1} \in[b] \bar{U}$, $b_{2} \in[b] \bar{V}$. Take $b_{1}^{\prime} \in\left\langle a, b_{1}\right\rangle, b_{2}^{\prime} \in\left\langle a, b_{2}\right\rangle$ such that $a V b_{1}^{\prime} U b_{1}, a U b_{2}^{\prime} V b_{2}$. The condition (P3) yields the existence of $t \geqslant b_{1}^{\prime}, b_{2}^{\prime}$ with $b_{2}^{\prime} V t U b_{1}^{\prime}$. Now $t \bar{U} b, t \bar{V} b$, hence $t=b$ by (P4). We have $b \geqslant a$.

Suppose $a \leqslant 1 b$. Then there exists $u \leqslant a, b$ satisfying $a V u U b$ and this implies that $[a] \bar{U} \geqslant[u] \bar{U}=[b] \bar{U},[a] \bar{V}=[u] \bar{V} \leqslant[b] \bar{V}$.

Finally, let $[a] \bar{U} \geqslant[b] \bar{U},[a] \bar{V} \leqslant[b] \bar{V}$. We have to show $a \leqslant{ }_{1} b$. The assumptions yield the existence of $a_{1} \in[a] \bar{U}, a_{2} \in[a] \bar{V}$ with $a_{2} \leqslant b \leqslant a_{1}$. Take $c \in\left\langle a_{2}, a_{1}\right\rangle$ satisfying $a_{2} V c U a_{1}$. Then $c=a$ by (P4). In view of $2.8 u=\inf \{a, b\}$ exists and $a V u U b$. The proof is complete.

3.

Let $\mathbb{A}=(A, \leqslant)$ be a connected partially ordered set, $\mathbb{A}^{\prime}=\left(A, \leqslant^{\prime}\right)$ another partially ordered set with the same underlying set and let $f$ be an isomorphism of Int $\mathbb{A}$ onto Int $\mathbb{A}^{\prime}$. The aim is to prove that $\mathbb{A}^{\prime}$ can be obtained in the way described in the preceding section. Define $f^{\prime}: A \rightarrow A$ by

$$
f^{\prime}(a)=b \Longleftrightarrow f(\langle a\rangle)=\langle b\rangle^{\prime}=\langle b\rangle
$$

( $\langle x, y\rangle^{\prime}$ will mean the set $\left.\left\{t \in A: x \leqslant^{\prime} t \leqslant \leqslant^{\prime} y\right\}\right)$. Evidently $f^{\prime}$ is a bijective mapping of $A$ onto $A$. Consider the following binary relations on $A: U=\{(x, y) \in A \times A: x \leqslant y$ and $\left.f^{\prime}(x) \leqslant f^{\prime} f^{\prime}(y)\right\} \cup\left\{(x, y) \in A \times A: x \geqslant y\right.$ and $\left.f^{\prime}(x) \geqslant f^{\prime}(y)\right\}, V=\{(x, y) \in$ $A \times A: x \leqslant y$ and $\left.f^{\prime}(x) \geqslant f^{\prime}(y)\right\} \cup\left\{(x, y) \in A \times A: x \geqslant y\right.$ and $\left.f^{\prime}(x) \leqslant f^{\prime}(y)\right\}$. Evidently $U, V$ satisfy the condition $(\mathrm{P} 1)$.

3.1. Lemma. Let $x, y \in A, x \leqslant y, f(\langle x, y\rangle)=\langle r, s\rangle^{\prime}$. Then $r=\inf \left\{f^{\prime}(x), f^{\prime}(y)\right\}$, $s=\sup \left\{f^{\prime}(x), f^{\prime}(y)\right\}$ in $\mathbb{A}^{\prime}$. 
Pro of. Since $\langle x, y\rangle=\sup \{\langle x\rangle,\langle y\rangle\}$, we have $\langle r, s\rangle^{\prime}=\sup \{f(\langle x\rangle), f(\langle y\rangle\}$. But $f(\langle x\rangle)=\left\langle f^{\prime}(x)\right\rangle, f(\langle y\rangle)=\left\langle f^{\prime}(y)\right\rangle$ so that $r=\inf \left\{f^{\prime}(x), f^{\prime}(y)\right\}, s=\sup \left\{f^{\prime}(x), f^{\prime}(y)\right\}$ in $\mathbb{A}^{\prime}$ by 1.1 .

Taking into account that $f^{-1}$ is also an isomorphism and $\left(f^{-1}\right)^{\prime}=\left(f^{\prime}\right)^{-1}$, we obtain:

3.2. Lemma. If $x, y \in A, x \leqslant y, f(\langle x, y\rangle)=\langle r, s\rangle^{\prime}, r=f^{\prime}(p), s=f^{\prime}(q)$, then $x=\inf \{p, q\}, y=\sup \{p, q\}$ in $\mathbb{A}$.

3.3. Lemma. The above defined $U, V$ fulfil (P2).

Proof. Let $x, y \in A, x \leqslant y$. The previous lemma guarantees the existence of such $p, q$ as we need. Now let $p_{1}, q_{1} \in\langle x, y\rangle$ also satisfy $p_{1} V x U q_{1} V y U p_{1}$. The relations $p_{1} V x, x \leqslant p_{1}$ imply $f^{\prime}(x) \geqslant f^{\prime}\left(p_{1}\right)$ while $y U p_{1}, p_{1} \leqslant y$ imply $f^{\prime}\left(p_{1}\right) \leqslant f^{\prime}(y)$. Hence $f^{\prime}\left(p_{1}\right) \leqslant \leqslant^{\prime} r$ by 3.1. Analogously $f^{\prime}\left(q_{1}\right) \geqslant{ }^{\prime} s$. On the other hand $\left\langle p_{1}\right\rangle,\left\langle q_{1}\right\rangle \subseteq\langle x, y\rangle$ yields $\left\langle f^{\prime}\left(p_{1}\right)\right\rangle,\left\langle f^{\prime}\left(q_{1}\right)\right\rangle \subseteq\langle r, s\rangle^{\prime}$ and consequently $r \leqslant f^{\prime}\left(p_{1}\right), f^{\prime}\left(q_{1}\right) \leqslant{ }^{\prime} s$. So we have $f^{\prime}\left(p_{1}\right)=r=f^{\prime}(p), f^{\prime}\left(q_{1}\right)=s=f^{\prime}(q)$, which implies $p_{1}=p, q_{1}=q$.

3.4. Lemma. Let $x, y \in A, x \leqslant y, x U y(x V y)$. Then for each $t \in\langle x, y\rangle$ we have $x U t U y(x V t V y)$.

Proof. We will prove, e.g., the part concerning $U$. Take any $t \in\langle x, y\rangle$. We have $\langle x, t\rangle \subseteq\langle x, y\rangle$, hence $f(\langle x, t\rangle) \subseteq f(\langle x, y\rangle)$. By the assumption $x U y$ we have $f^{\prime}(x) \leqslant f^{\prime}(y)$ and using 3.1 we obtain $f(\langle x, y\rangle)=\left\langle f^{\prime}(x), f^{\prime}(y)\right\rangle^{\prime}$. Let $f(\langle x, t\rangle)=$ $\langle a, b\rangle^{\prime}$. Then $\langle a, b\rangle^{\prime} \subseteq\left\langle f^{\prime}(x), f^{\prime}(y)\right\rangle^{\prime}$, which implies $f^{\prime}(x) \leqslant{ }^{\prime} a$. On the other hand $a=\inf \left\{f^{\prime}(x), f^{\prime}(t)\right\}$ in $\mathbb{A}^{\prime}$ by 3.1 , so that $a \leqslant^{\prime} f^{\prime}(t)$. Summarizing we obtain $f^{\prime}(x) \leqslant f^{\prime}(t)$. We have $x U t$. The relation $t U y$ can be shown analogously.

3.5. Lemma. The above defined $U, V$ fulfil $(\mathrm{P} 3)$ and $\left(\mathrm{P} 3^{\prime}\right)$.

Proof. We are going to show that (P3) holds. The condition (P3') can be verified analogously. Let $u \leqslant x, y, x V u U y$. Then $f^{\prime}(x) \leqslant f^{\prime}(u) \leqslant f^{\prime}(y)$. Let $\left\langle f^{\prime}(x), f^{\prime}(y)\right\rangle^{\prime}=f(\langle a, b\rangle$. Using 3.1 and 3.2 we obtain $a=\inf \{x, y\}, b=\sup \{x, y\}$ in $\mathbb{A}, f^{\prime}(x)=\inf \left\{f^{\prime}(a), f^{\prime}(b)\right\}, f^{\prime}(y)=\sup \left\{f^{\prime}(a), f^{\prime}(b)\right\}$ in $\mathbb{A}^{\prime}$. Since $u$ is a lower bound of $\{x, y\}$, we infer $u \leqslant a$. Now $a \in\langle u, x\rangle \cap\langle u, y\rangle$, hence $u U a$ and simultaneously $u V a$ by 3.4. Since $u \leqslant a$, we have $f^{\prime}(u) \leqslant f^{\prime}(a)$ and simultaneously $f^{\prime}(u) \geqslant^{\prime} f^{\prime}(a)$. Then $f^{\prime}(u)=f^{\prime}(a)$ and consequently $u=a$. We have proved $u=\inf \{x, y\}$. It remains to show $y V b U x$. Since $b \geqslant x, y$, we have to verify $f^{\prime}(x) \leqslant^{\prime} f^{\prime}(b) \leqslant f^{\prime}(y)$, but this is evident. 
Summarizing, having an isomorphism $f: \operatorname{Int} \mathbb{A} \rightarrow \operatorname{Int} \mathbb{A}^{\prime}$, we can construct binary relations $U, V$ on $A$ satisfying (P1)-(P3'). Further, using 2.14, we obtain a partially ordered set $\mathbb{A}_{1}$ such that Int $\mathbb{A}$ and Int $\mathbb{A}_{1}$ are isomorphic. The following theorem makes clear the relation between $\mathbb{A}^{\prime}$ and $\mathbb{A}_{1}$.

3.6. Theorem. Let $\mathbb{A}=(A, \leqslant)$ be a connected partially ordered set, $\mathbb{A}^{\prime}=$ $\left(A, \leqslant^{\prime}\right)$ any partially ordered set such that $\operatorname{Int} \mathbb{A}^{\prime}$ is isomorphic to $\operatorname{Int} \mathbb{A}$. If $U, V$ are defined with the aid of an isomorphism $f: \operatorname{Int} \mathbb{A} \rightarrow \operatorname{Int} \mathbb{A}^{\prime}$ as above, then the partially ordered set $\mathbb{A}_{1}$ corresponding to $U, V$ in the sense of 2.14 is isomorphic to $\mathbb{A}^{\prime}$.

P r o of. We are going to show that the mapping $f^{\prime}$ belonging to the isomorphism $f: \operatorname{Int} \mathbb{A} \rightarrow \operatorname{Int} \mathbb{A}^{\prime}$ is an isomorphism of $\mathbb{A}_{1}$ onto $\mathbb{A}^{\prime}$. It is sufficient to prove that $x \leqslant_{1} y$ if and only if $f^{\prime}(x) \leqslant^{\prime} f^{\prime}(y)$. Let $x \leqslant \leqslant_{1} y$. Then there exist $u \leqslant x, y$ satisfying $x V u U y$. Using the definition of $U, V$ we obtain $f^{\prime}(x) \leqslant f^{\prime}(u) \leqslant f^{\prime}(y)$. Conversely, let $f^{\prime}(x) \leqslant^{\prime} f^{\prime}(y)$. Considering $\langle a, b\rangle=f^{-1}\left(\left\langle f^{\prime}(x), f^{\prime}(y)\right\rangle^{\prime}\right)$ and using 3.2, 3.1, we obtain $a=\inf \{x, y\}, x V a U y$. Hence $x \leqslant_{1} y$. The proof is complete.

Notice that if $(A, \leqslant),(A, \leqslant 1)$ are as in 2.14 , then Int $(A, \leqslant)$, Int $(A, \leqslant 1)$ are not only isomorphic, but even identical as systems of subsets of $A$. Moreover, every $\left(A, \leqslant^{\prime}\right)$ satisfying that $\operatorname{Int}\left(A, \leqslant^{\prime}\right)$ is identical with $\operatorname{Int}(A, \leqslant)$ can be obtained in this way by 3.6.

To have 1.2 completely proved, we add:

3.7. Theorem. Let $\mathbb{A}=\mathbb{C} \times \mathbb{D}$ be a connected partially ordered set, $\mathbb{A}^{\prime}=\mathbb{C}^{\delta} \times \mathbb{D}$, let $f: \operatorname{Int} \mathbb{A} \rightarrow \operatorname{Int} \mathbb{A}^{\prime}$ be defined by

$$
\left.f\left(\left\langle c_{1}, d_{1}\right),\left(c_{2}, d_{2}\right)\right\rangle\right)=\left\langle\left(c_{2}, d_{1}\right),\left(c_{1}, d_{2}\right)\right\rangle^{\prime}
$$

Then $f$ is an isomorphism and if $U, V$ are defined with the aid of $f$ as at the beginning of this section, they satisfy the conditions (P4) and (P5).

Pro of. The assertion that $f$ is an isomorphism is evident. Obviously, $f^{\prime}$ is the identity mapping, so that $\left(c_{1}, d_{1}\right) U\left(c_{2}, d_{2}\right)$ means that $c_{1}=c_{2}$ and simultaneously $d_{1}, d_{2}$ are comparable while $\left(c_{1}, d_{1}\right) V\left(c_{2}, d_{2}\right)$ means that $c_{1}, c_{2}$ are comparable and $d_{1}=d_{2}$.

Now let

$$
\begin{aligned}
& (c, d)=\left(c_{1}, d_{1}\right) U\left(c_{2}, d_{2}\right) U \ldots U\left(c_{n}, d_{n}\right)=\left(c^{\prime}, d^{\prime}\right), \\
& (c, d)=\left(c_{1}^{\prime}, d_{1}^{\prime}\right) V\left(c_{2}^{\prime}, d_{2}^{\prime}\right) V \ldots V\left(c_{m}^{\prime}, d_{m}^{\prime}\right)=\left(c^{\prime}, d^{\prime}\right) .
\end{aligned}
$$


Then $c=c_{1}=c_{2}=\ldots=c^{\prime}$ and $d=d_{1}^{\prime}=d_{2}^{\prime}=\ldots=d^{\prime}$, so that $(c, d)=\left(c^{\prime}, d^{\prime}\right)$.

Finally, taking any $(c, d),\left(c^{\prime}, d^{\prime}\right) \in C \times D$ and using the fact that $\mathbb{A}$ is connected so that $\mathbb{C}, \mathbb{D}$ are connected, too, we can find $c_{1}, \ldots, c_{n} \in C, d_{1}, \ldots, d_{m} \in D$ such that $c_{1}=c, c_{n}=c^{\prime}, d_{1}=d, d_{m}=d^{\prime}, c_{i}$ is comparable with $c_{i+1}$ for each $i \in\{1, \ldots, n-1\}$ and $d_{j}$ is comparable with $d_{j+1}$ for each $j \in\{1, \ldots, m-1\}$. Then we have $(c, d)=$ $\left(c_{1}, d_{1}\right) U\left(c_{1}, d_{2}\right) U \ldots U\left(c_{1}, d_{m}\right)=\left(c_{1}, d^{\prime}\right) V\left(c_{2}, d^{\prime}\right) V \ldots V\left(c_{n}, d^{\prime}\right)=\left(c^{\prime}, d^{\prime}\right)$. The proof is complete.

Notice that the mapping $\Phi$ in Theorem 1.2 is not one-to-one, in general. For example, if $\mathbb{A}$ is a selfdual partially ordered set, then both $U_{1}=\{(x, y) \in A \times A: x \nVdash$ $y\}, V_{1}=\{(x, x): x \in A\}$ and $U_{2}=\{(x, x): x \in A\}, V_{2}=\{(x, y) \in A \times A: x \nmid y\}$ lead to the same isomorphism class of partially ordered sets. In this connection, a natural question arises: under what conditions two couples $U_{1}, V_{1}$ and $U_{2}, V_{2}$ of binary relations on $A$ satisfying (P1)-(P3') give the same isomorphism class of partially ordered sets.

Having a bijection $\alpha$ of $A$ onto $A$, binary relations $U_{1}, V_{1}$ on $A$ satisfying (P1)$\left(\mathrm{P}^{\prime}\right)$ and the corresponding partial order $\leqslant 1$ (in the sense of 2.10 ), consider the following conditions:

(C1) $\alpha(x) \leqslant \alpha(y) \Longrightarrow$ there exists a unique couple of elements $p, q \in A$ satisfying $\alpha(x) \leqslant \alpha(p), \alpha(q) \leqslant \alpha(y), p \leqslant{ }_{1} x, y \leqslant 1 q$

(C2) $p \leqslant 1 q \Longrightarrow \inf \{\alpha(p), \alpha(q)\}, \sup \{\alpha(p), \alpha(q)\}$ exist and

$$
\begin{aligned}
& x=\alpha^{-1}(\inf \{\alpha(p), \alpha(q)\}), \\
& y=\alpha^{-1}(\sup \{\alpha(p), \alpha(q)\})
\end{aligned}
$$

are the only elements of $A$ satisfying $\alpha(x) \leqslant \alpha(p), \alpha(q) \leqslant \alpha(y), p \leqslant 1 x$, $y \leqslant{ }_{1} q$.

3.8. Theorem. Let $U_{1}, V_{1}$ and $U_{2}, V_{2}$ be two couples of binary relations on $A$ satisfying $(\mathrm{P} 1)-\left(\mathrm{P}^{\prime}\right)$, let $\leqslant_{1}$ and $\leqslant_{2}$ be the corresponding partial orders (in the sense of 2.10). If $\alpha$ is an isomorphism of $\left(A, \leqslant_{1}\right)$ onto $\left(A, \leqslant_{2}\right)$, then

(1) $\alpha$ fulfils (C1), (C2) and

(2) $U_{2}=\left\{(x, y) \in A \times A: x \leqslant y\right.$ and $\left.\alpha^{-1}(x) \leqslant{ }_{1} \alpha^{-1}(y)\right\} \cup\{(x, y) \in A \times A: x \geqslant y$ and $\left.\alpha^{-1}(x) \geqslant{ }_{1} \alpha^{-1}(y)\right\}, V_{2}=\left\{(x, y) \in A \times A: x \leqslant y\right.$ and $\alpha^{-1}(x) \geqslant_{1}$ $\left.\alpha^{-1}(y)\right\} \cup\left\{(x, y) \in A \times A: x \geqslant y\right.$ and $\left.\alpha^{-1}(x) \leqslant{ }_{1} \alpha^{-1}(y)\right\}$.

Proof. We start with (2). Obviously $U_{2}=\{(x, y) \in A \times A: x \leqslant y$ and $\left.x \leqslant \leqslant_{2} y\right\} \cup\left\{(x, y) \in A \times A: x \geqslant y\right.$ and $\left.x \geqslant_{2} y\right\}$. Since $r \leqslant_{2} s(r, s \in A)$ is equivalent to $\alpha^{-1}(r) \leqslant 1 \alpha^{-1}(s)$, we have what we need for $U_{2}$. As to $V_{2}$, we proceed analogously. 
Now let $\alpha(x) \leqslant \alpha(y)$. Then there exists a unique couple of $\alpha(p), \alpha(q) \in$ $\langle\alpha(x), \alpha(y)\rangle$ with $\alpha(p) V_{2} \alpha(x) U_{2} \alpha(q) V_{2} \alpha(y) U_{2} \alpha(p)$ by (P2). The latter is equivalent to $\alpha(p) \leqslant_{2} \alpha(x), \alpha(y) \leqslant_{2} \alpha(q)$ and this holds if and only if $p \leqslant_{1}, x, y \leqslant 1 q$.

To prove (C2), take $p \leqslant{ }_{1} q$. Then $\alpha(p) \leqslant{ }_{2} \alpha(q)$ and there exists $\alpha(x) \leqslant \alpha(p), \alpha(q)$ satisfying $\alpha(p) V_{2} \alpha(x) U_{2} \alpha(q)$. Using (P3) we obtain that $\alpha(x)=\inf \{\alpha(p), \alpha(q)\}$, $\alpha(y)=\sup \{\alpha(p), \alpha(q)\}$ exists and we have $\alpha(q) V_{2} \alpha(y) U_{2} \alpha(p)$. The latter means $\alpha(p) \leqslant_{2} \alpha(y) \leqslant_{2} \alpha(q)$, which is equivalent to $p \leqslant_{1} y \leqslant_{1} q$. The relation $p \leqslant_{1} x \leqslant_{1} q$ follows from $\alpha(p) V_{2} \alpha(x) U_{2} \alpha(q)$. Now having $x_{1}, y_{1}$ satisfying $\alpha\left(x_{1}\right) \leqslant \alpha(p), \alpha(q) \leqslant$ $\alpha\left(y_{1}\right), p \leqslant 1 x_{1}, y_{1} \leqslant_{1} q$, it is easy to see that $\alpha(p) V_{2} \alpha\left(x_{1}\right) U_{2} \alpha(q) V_{2} \alpha\left(y_{1}\right) U_{2} \alpha(p)$, which yields $\alpha\left(x_{1}\right)=\inf \{\alpha(p), \alpha(q)\}, \alpha\left(y_{1}\right)=\sup \{\alpha(p), \alpha(q)\}$ by (P3) and (P3'). But then $\alpha\left(x_{1}\right)=\alpha(x), \alpha\left(y_{1}\right)=\alpha(y)$ and consequently $x_{1}=x, y_{1}=y$.

The proof is complete.

Conversely, we have:

3.9. Theorem. Let $U_{1}, V_{1}$ be a couple of binary relations on $A$ satisfying $(\mathrm{P} 1)-$ $\left(\mathrm{P} 3^{\prime}\right)$ and let $\alpha$ be any bijection of $A$ onto $A$ satisfying $(\mathrm{C} 1),(\mathrm{C} 2)$. Taking $U_{2}, V_{2}$ as in (2) of 3.8, they satisfy the conditions (P1)-(P3') and $\alpha$ is an isomorphism of $\left(A, \leqslant_{1}\right)$ onto $\left(A, \leqslant_{2}\right)\left(\leqslant_{1}\right.$ and $\leqslant_{2}$ are the partial orders corresponding to $U_{1}, V_{1}$ and $U_{2}, V_{2}$, respectively, in the sense of 2.10$)$.

Pro of. The relations $U_{2}, V_{2}$ satisfy (P1) trivially. To prove (P2), let $x^{\prime}, y^{\prime} \in A$, $x^{\prime} \leqslant y^{\prime}$. Take $x, y \in A$ with $\alpha(x)=x^{\prime}, \alpha(y)=y^{\prime}$. The condition (C1) yields the existence of a unique couple of elements $p, q \in A$ satisfying $\alpha(x) \leqslant \alpha(p), \alpha(q) \leqslant \alpha(y)$, $p \leqslant_{1} x, y \leqslant_{1} q$. Set $\alpha(p)=p^{\prime}, \alpha(q)=q^{\prime}$. Then $p^{\prime}, q^{\prime}$ are the only elements of the interval $\left\langle x^{\prime}, y^{\prime}\right\rangle$ with $p^{\prime} V_{2} x^{\prime} U_{2} q^{\prime} V_{2} y^{\prime} U_{2} p^{\prime}$.

We are going to prove (P3). The proof of (P3') would be analogous. Let $u^{\prime} \leqslant p^{\prime}, q^{\prime}$, $p^{\prime} V_{2} u^{\prime} U_{2} q^{\prime}$. Take $u, p, q$ with $\alpha(u)=u^{\prime}, \alpha(p)=p^{\prime}, \alpha(q)=q^{\prime}$. Using the definition of $U_{2}, V_{2}$ we obtain $p \leqslant_{1} u \leqslant_{1} q$. Now (C2) ensures the existence of $\inf \{\alpha(p), \alpha(q)\}$ and $\sup \{\alpha(p), \alpha(q)\}$, together with $u^{\prime}=\inf \{\alpha(p), \alpha(q)\}$. It remains to show that $q^{\prime} V_{2} \sup \left\{p^{\prime}, q^{\prime}\right\} U_{2} p^{\prime}$, which is equivalent to $p \leqslant_{1} \alpha^{-1}\left(\sup \left\{p^{\prime}, q^{\prime}\right\}\right) \leqslant 1$. But this holds by $(\mathrm{C} 2)$.

Finally, we have to prove that having any $x, y \in A, x \leqslant 1$ is equivalent to $\alpha(x) \leqslant 2$ $\alpha(y)$. Let $x \leqslant_{1} y$. Then there exists $u \in\langle x, y\rangle_{1} \operatorname{such}$ that $\alpha(u)=\inf \{\alpha(x), \alpha(y)\}$ by (C2). Now $\alpha(u) \leqslant \alpha(x)$ together with $u \geqslant_{1} x$ yields $\alpha(u) V_{2} \alpha(x)$, while $\alpha(u) \leqslant$ $\alpha(y), u \leqslant 1 \quad y$ implies $\alpha(u) U_{2} \alpha(y)$. Consequently $\alpha(x) \leqslant 2 \alpha(y)$. Conversely, let $\alpha(x) \leqslant 2 \alpha(y)$. Then there exists $\alpha(t) \leqslant \alpha(x), \alpha(y)$ with $\alpha(x) V_{2} \alpha(t) U_{2} \alpha(y)$. So we have $x \leqslant_{1} t \leqslant_{1} y$ and the proof is complete.

It is easy to see that if $\alpha$ is an automorphism or a dual automorphism of $\mathbb{A}$, then $\alpha$ satisfies the conditions $(\mathrm{C} 1),(\mathrm{C} 2)$ for any $U_{1}, V_{1}$ fulfilling $(\mathrm{P} 1)-\left(\mathrm{P} 3^{\prime}\right)$ and 
the corresponding order $\leqslant_{1}$. But a bijection $\alpha$ fulfilling $(\mathrm{C} 1)$, (C2) need not be an isomorphism or a dual isomorphism, as the following example shows:

3.10. Example. Let $\mathbb{A}$ be as in Fig. 4. Let $U_{1}=\{(a, b) \in A \times A: a \nmid b\}, V_{1}=$ $\{(t, t): t \in A\}, U_{2}=V_{1}, V_{2}=U_{1}, U_{3}=\{(u, y),(y, u),(x, v),(v, x)\} \cup\{(t, t): t \in A\}$, $V_{3}=\{(x, u),(u, x),(y, v),(v, y)\} \cup\{(t, t): t \in A\}, U_{4}=V_{3}, V_{4}=U_{3}$. It is easy to see that all couples satisfying $(\mathrm{P} 1)-\left(\mathrm{P} 3^{\prime}\right)$ are those of $U_{1}, V_{1}, U_{2}, V_{2}, U_{3}, V_{3}$ and $U_{4}, V_{4}$. But each of them yields the same isomorphism class of partially ordered sets with the interval system isomorphic to Int $\mathbb{A}$. E.g., the mapping $\alpha$ such that $\alpha(u)=v$, $\alpha(v)=u, \alpha(x)=x, \alpha(y)=y$ mediates the transition from $U_{1}, V_{1}$ to $U_{2}, V_{2}$, while $\Psi$ defined by $\Psi(u)=y, \Psi(y)=v, \Psi(v)=x, \Psi(x)=u$ is an intermediary between $U_{1}, V_{1}$ and $U_{4}, V_{4}$.

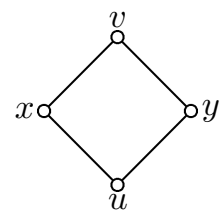

Fig. 4

The following question remains open:

Let $\mathbb{A}_{1} \times \mathbb{A}_{2}$ be a direct decompositon of $\mathbb{A}$. Consider the class of all partially ordered sets isomorphic to $\mathbb{A}_{1}^{\delta} \times \mathbb{A}_{2}$. Does every pre-image $(U, V)$ of this class under $\Phi$ satisfy (P4), (P5)? In particular, if the class $\Phi((U, V))$ consists of all partially ordered sets isomorphic to $\mathbb{A}\left(\right.$ or $\left.\mathbb{A}^{\delta}\right)$, does $(U, V)$ satisfy $(\mathrm{P} 4),(\mathrm{P} 5)$ ?

4.

In this section we will apply the foregoing results to the case of a directed partially ordered set.

4.1. Lemma. Let $\mathbb{A}=(A, \leqslant)$ be a directed partially ordered set, $U, V$ binary relations on $A$ satisfying $(\mathrm{P} 1)-\left(\mathrm{P} 3^{\prime}\right)$. Then $U, V$ satisfy also $(\mathrm{P} 4)$ and $(\mathrm{P} 5)$.

P r o of. Let $a=a_{1} U a_{2} U \ldots U a_{n}=a^{\prime}, a=a_{1}^{\prime} V a_{2}^{\prime} V \ldots V a_{m}^{\prime}=a^{\prime}$. Take a lower bound $x$ and an upper bound $y$ of the set $\left\{a_{1}, \ldots, a_{n}, a_{1}^{\prime} \ldots, a_{m}^{\prime}\right\}$ and elements $p, q$ as in (P2). Using 2.9 we get $\inf \{p, a\}=\inf \left\{p, a_{1}\right\}=\inf \left\{p, a_{2}\right\}=\ldots=\inf \left\{p, a^{\prime}\right\}$ and analogously $\inf \{a, q\}=\inf \left\{a^{\prime}, q\right\}$. But then $a=a^{\prime}$ by 2.8 .

Further let $a, a^{\prime} \in A$. Take a lower bound $x$ and an upper bound $y$ of $\left\{a, a^{\prime}\right\}$ and $p, q$ as in (P2). Then $p_{1}=\inf \{p, a\}, q_{1}^{\prime}=\inf \left\{a^{\prime}, q\right\}$ satisfy $p V p_{1} U a, a^{\prime} V q_{1}^{\prime} U q$, $p_{1} V x U q_{1}^{\prime}$. (P3) ensures the existence of $t=\sup \left\{p_{1}, q_{1}^{\prime}\right\}$ with $q_{1}^{\prime} V t U p_{1}$. Hence $a U p_{1} U t V q_{1}^{\prime} V a^{\prime}$, completing the proof. 
Using 1.2 we immediately get:

4.2. Theorem. Let $\mathbb{A}$ be a directed partially ordered set. If $\mathbb{B}$ is a partially ordered set with Int $\mathbb{B}$ isomorphic to $\operatorname{Int} \mathbb{A}$, then there exist partially ordered sets $\mathbb{C}$, $\mathbb{D}$ such that $\mathbb{A}$ is isomorphic to $\mathbb{C} \times \mathbb{D}$ and $\mathbb{B}$ is isomorphic to $\mathbb{C}^{\delta} \times \mathbb{D}$.

The converse is evident, so we have:

4.3. Corollary. Let $\mathbb{A}$ be a directed partially ordered set, $\mathbb{B}$ any partially ordered set. The following conditions are equivalent:

(i) Int $\mathbb{B}$ is isomorphic to $\operatorname{Int} \mathbb{A}$,

(ii) there exist partially ordered sets $\mathbb{C}, \mathbb{D}$ such that $\mathbb{A}$ is isomorphic to $\mathbb{C} \times \mathbb{D}$ and $\mathbb{B}$ is isomorphic to $\mathbb{C}^{\delta} \times \mathbb{D}$.

Since lattices are directed partially ordered sets, we obtain Theorem 1 of [11] as a consequence of 4.3. Let us notice that if $\mathbb{A}$ is a lattice and $\mathbb{B}$ is a partially ordered set with Int $\mathbb{B}$ isomorphic to Int $\mathbb{A}$, then $\mathbb{B}$ is also a lattice, as 4.3 shows.

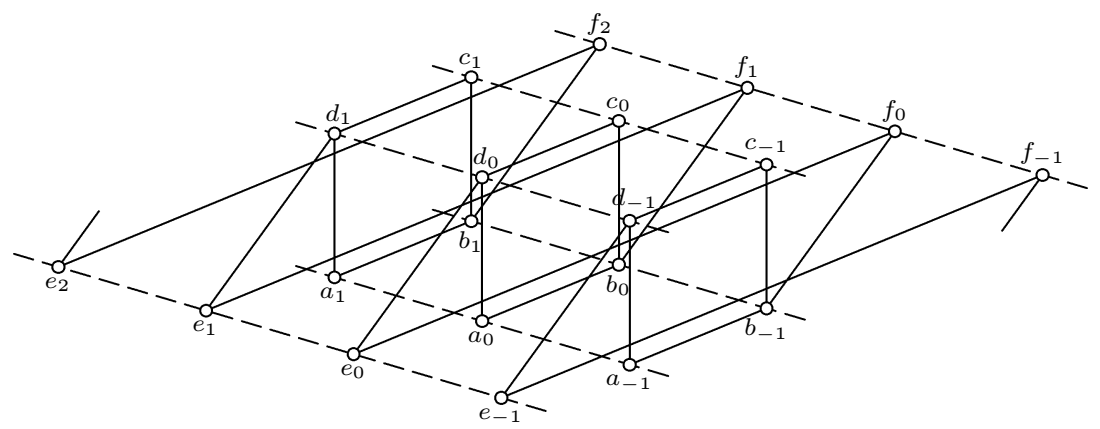

Fig. 5

Without the assumption that $\mathbb{A}$ is directed, the assertion of 4.3 is false. To show this, consider $\mathbb{A}$ as in Fig. 5. Let $U$ and $V$ be the relations marked out by the full and dashed lines, respectively. It is easy to see that $U, V$ fulfil the conditions $(\mathrm{P} 1)-\left(\mathrm{P} 3^{\prime}\right)$ and $(\mathrm{P} 5)$, but $(\mathrm{P} 4)$ is not satisfied (e.g. $e_{0} V e_{1}$ and simultaneously $e_{0} U d_{0} U a_{0} U b_{0} U f_{1} U e_{1}$ holds). Taking the corresponding $\mathbb{A}_{1}$ (in the sense of 2.10) as $\mathbb{B}$, depicted in Fig. 6, it fulfils (i) of 4.3, while it fails to satisfy (ii) of 4.3. 


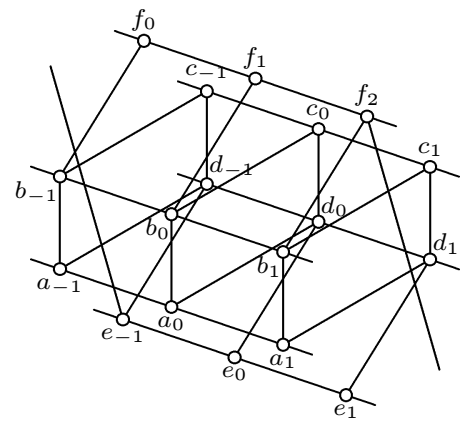

Fig. 6

\section{References}

[1] V. I. Igošin: Selfduality of lattices of intervals of finite lattices. Inst. Matem. Sibir. Otdel. AN SSSR, Meždunarodnaja konferencija po algebre posvjaščennaja pamjati A. I. Mal’ceva. Tezisy dokladov po teoriji modelej i algebraičeskich sistem, Novosibirsk 1989, p. 48. (In Russian.)

[2] V. I. Igošin: Lattices of intervals and lattices of convex sublattices of lattices. Uporjadočennyje množestva i rešotki. Saratov 6 (1990), 69-76. (In Russian.)

[3] V. I. Igošin: Identities in interval lattices of lattices. Coll. Math. Soc. J. Bolyai 33 (Contributions to Lattice Theory), Szeged 1980 (1983), 491-501.

[4] V. I. Igošin: On lattices with restriction on their intervals. Coll. Math. Soc. J. Bolyai 43 (Lectures in Universal Algebra), Szeged 1983 (1986), 209-216.

[5] V. I. Igošin: Algebraic characteristic of lattices of intervals. Uspechi matem. nauk 40 (1985), 205-206. (In Russian.)

[6] V. I. Igošin: Semimodularity in lattices of intervals. Math. Slovaca 38 (1988), 305-308.

[7] J. Jakubik: Selfduality of the system of intervals of a partially ordered set. Czechoslov. Math. J. 41 (1991), 135-140.

[8] J. Jakubik, J. Lihová: Systems of intervals of partially ordered sets. Math. Slovaca, to appear.

[9] M. Kolibiar: Intervals, convex sublattices and subdirect representations of lattices. Universal Algebra and Applications, Banach Center Publications, Vol. 9, Warsaw 1982, 335-339.

[10] J. Lihová: Posets having a selfdual interval poset. Czechoslovak Math. J. 44 (1994), 523-533.

[11] V. Slavik: On lattices with isomorphic interval lattices. Czechoslovak Math. J. 35 (1985), 550-554.

Author's address: Prírodovedecká fakulta UPJŠ, 04154 Košice, Jesenná 5, Slovakia. 